\title{
Erratum to: Preliminary data of VEGF-A and VEGFR-2 \\ polymorphisms as predictive factors of radiological response and clinical outcome in iodine-refractory differentiated thyroid cancer treated with sorafenib
}

\author{
Vincenzo Marotta ${ }^{1}$ - Concetta Sciammarella ${ }^{1}$ Mario Capasso ${ }^{2} \cdot$ Alessandro Testori $^{2}$. \\ Claudia Pivonello ${ }^{3}$ Maria Grazia Chiofalo ${ }^{4} \cdot$ Rosario Pivonello $^{3} \cdot$ Luciano Pezzullo $^{4}$. \\ Gerardo Botti $^{5} \cdot$ Annamaria Colao $^{3} \cdot$ Antongiulio Faggiano $^{4}$
}

Published online: 27 February 2017

(C) Springer Science+Business Media New York 2017

\section{Erratum to: Endocrine} DOI 10.1007/s12020-016-1200-6

In the original publication, the Acknowledgements section was missing.
Acknowledgements We would like to acknowledge Umberto Veronesi Foundation for granting V.M. with Postdoctoral Fellowship year-2017 Award.

The online version of the original article can be found at doi:10.1007/ s12020-016-1200-6.

Vincenzo Marotta

vinc.endo@libero.it

IOS \& COLEMAN Srl, Naples, Italy

2 CEINGE Biotecnologie Avanzate, Federico II University, Naples, Italy

3 Department of Clinical Medicine and Surgery, Federico II University, Naples, Italy

4 Thyroid and Parathyroid Surgery Unit, Istituto Nazionale per lo Studio e la Cura dei Tumori-IRCCS "Fondazione G. Pascale", Naples, Italy

5 Department of Pathology, Istituto Nazionale per lo Studio e la Cura dei Tumori-IRCCS "Fondazione G. Pascale", Naples, Italy 